\title{
Identifiability, stability and reconstruction results of point sources by boundary measurements in heteregeneous trees
}

\author{
Serge NICAISE ${ }^{(1)}$ and Ouahiba ZAïR ${ }^{(2)}$ \\ (1) Université de Valenciennes et du Hainaut Cambrésis \\ MACS, ISTV \\ F-59313 - Valenciennes Cedex 9, France \\ snicaise@univ-valenciennes.fr \\ (2) Université des Sciences et de la Technologie $H$. Boumediene \\ Institut de Mathématiques \\ El-Alia, B.P. 32 \\ Bab Ezzouar, Alger-Algeria
}

Recibido: 4 de Marzo de 2002

Aceptado: 8 de Julio de 2002

\begin{abstract}
We consider the inverse problem of determining point wave sources in heteregeneous trees, extensions of one-dimensional stratified sets. We show that the Neumann boundary observation on a part of the lateral boundary determines uniquely the point sources if the time of observation is large enough. We further establish a conditional stability and give a reconstructing scheme.
\end{abstract}

2000 Mathematics Subject Classification: 35R30, 35J25.

Key words: Inverse problem

\section{Introduction}

Various models of multiple-link flexible structures, consisting of finitely many interconnected flexible elements, like strings, beams, plates, shells or combinations of them, have been described recently in $[22,16,24,25,13,12]$. The problem of controllability or stabilization of such structures is an expanding field. For control results, let us quote the works of Lagnese-Leugering-Schmidt [34, 23, 24] and of Dager-Zuazua [18] for 1-d. networks; the works of Puel-Zuazua [33], Lagnese [21] and the first author $[30,31,32]$ for multidimensional structures. For stabilization results, we may cite the 
results of Chen and coauthors $[13,14,15]$, of Conrad [17] and of Lagnese-LeugeringSchmidt [23].

To our knowledge inverse problems related to the above control problems on such structures are not considered at all, contrary to the case of homogeneous structures (like one string, one beam, one membrane, one plate, etc...) for which such problems have been recently developed by Yamamoto and Bruckner [36, 10, 11, 37]. Therefore the goal of this paper is first to consider the simplest multi-dimensional problem: namely the wave equation on one-dimensional trees with some point sources (linear combination of delta functions, see below for the details) and secondly to extend the results from $[9,10]$ obtained for the real interval $] 0,1[$ to this system. In the case of serially connected strings this problem may be seen as a simplification of a model of earthquakes [1].

The questions in such problems are to determine the number of point sources, the location of these points and their intensity from boundary measurements. As usual the three main steps are the uniqueness (unique solvability of the problem), the stability (small perturbations of the boundary measurements give rise to small perturbations of the sources) and finally the reconstruction (build appropriate processes in order to find a good approximation of the unknowns). We answer to these questions by adapting some results from $[9,10,36]$ to our system. The main ingredients are the spectral analysis of the Laplace equation on networks (see [2, 4, 5, 8, 24, 27, 28] and the references cited there), some controllability results on such structures [34, 24] and finally appropriate properties of some integral operators [36, 10]. Since the eigenvalues and eigenvectors of the Laplace equation on networks are not explicitly known, our reconstruction process is different from the one in [10] and is more close to the one in $[36]$.

In [11] the authors consider an interior observation for the determination of the point sources in $] 0,1[$. The extension of this kind of considerations to $1-\mathrm{d}$. trees seems to be unrealistic since the point $\eta$ of observation in [11] is assumed to be an irrational algebraic number. This assumption guarantees that $\eta$ is never a zero of any eigenvector of the Laplace operator on ]0,1[ with Dirichlet boundary conditions. For 1-d. trees such a point is difficult to determine in practice.

The determination of $L^{2}$-source functions from boundary measurements on 1-d. trees may be obtained using the method developed in $[35,36]$ and the arguments used below.

The paper is organized as follows: In section 2 we recall some notations and definitions concerning 1-d. networks and introduce the (spatial) operator, namely a second order operator on each edge with some transmision conditions at interior nodes and Dirichlet boundary conditions at exterior nodes. We further show the wellposedness of the wave equation with point sources. Some observability estimates and hidden regularities are obtained in section 3 and are actually based on an identity with multiplier and its consequences namely the direct and inverse inequalities for the wave equation in a tree. Section 4 is devoted to the proof of the uniqueness result and 
is based on the previous observability estimates and some properties of an integral operator between different Sobolev spaces. The stability is deduced in section 5 and finally the reconstruction is detailed in section 6 .

\section{Acknowledgements}

We express our gratitude to the Université des Sciences et de la Technologie H. Boumediene for the financial support of the second named author and the laboratory MACS from the Université de Valenciennes for the kind hospitality of the second named author during various stays at Valenciennes.

\section{Preliminaries}

We first recall the notion of $C^{\nu}$-networks, $\nu \in \mathbb{N}$, which is simply those of [6], we refer to $[2,4,5,7,8]$ for more details.

All graphs considered here are non empty, finite and simple. Let $\Gamma$ be a connected topological graph imbedded in $\mathbb{R}^{m}, m \in \mathbb{N}^{*}$, with $n$ vertices $E=\left\{E_{i}: 1 \leq i \leq n\right\}$ and $N$ edges $K=\left\{k_{j}: 1 \leq j \leq N\right\}$. Each edge $k_{j}$ is a Jordan curve in $\mathbb{R}^{m}$ and is assumed to be parametrized by its arc length parameter $x_{j}$, such that the parametrizations

$$
\pi_{j}:\left[0, l_{j}\right] \rightarrow k_{j}: x_{j} \mapsto \pi_{j}\left(x_{j}\right)
$$

is $\nu$-times differentiable, i.e., $\pi_{j} \in C^{\nu}\left(\left[0, l_{j}\right], \mathbb{R}^{m}\right)$ for all $1 \leq j \leq N$.

We now define the $C^{\nu}$-network $G$ associated with $\Gamma$ as the union

$$
G=\cup_{j=1}^{N} k_{j} .
$$

The valency of each vertex $E_{i}$ is denoted by $\gamma\left(E_{i}\right)$. We distinguish two types of vertices: ramified (or interior) vertices int $E=\left\{E_{i} \in E: \gamma\left(E_{i}\right)>1\right\}$ and boundary (or exterior) vertices $\partial E=\left\{E_{i} \in E: \gamma\left(E_{i}\right)=1\right\}$. For shortness, we later on denote by $I_{\text {ext }}=\left\{i \in\{1, \cdots, n\}: \gamma\left(E_{i}\right)=1\right\}$ and $I_{\text {int }}=\{1, \cdots, n\} \backslash I_{\text {ext }}$. For each vertex $E_{i}$, we also denote by $N_{i}=\left\{j \in\{1, \ldots, N\}: E_{i} \in k_{j}\right\}$ the set of edges adjacent to $E_{i}$. Note that if $E_{i} \in \partial E$ then $N_{i}$ is a singleton that we write $\left\{j_{i}\right\}$. For each vertex $E_{i}$ and $j \in E_{i}$, we further denote by

$$
\nu_{j}\left(E_{i}\right)=\left\{\begin{array}{lll}
1 & \text { if } & \pi_{j}\left(l_{j}\right)=E_{i}, \\
-1 & \text { if } & \pi_{j}(0)=E_{i},
\end{array}\right.
$$

the normal vector in $k_{j}$ at $E_{i}$.

For a function $u: G \rightarrow \mathbb{R}$, we set $u_{j}=u \circ \pi_{j}:\left[0, l_{j}\right] \rightarrow \mathbb{R}$, its "restriction" to the edge $k_{j}$. We further use the abbreviations:

$$
\begin{aligned}
u_{j}\left(E_{i}\right) & =u_{j}\left(\pi_{j}^{-1}\left(E_{i}\right)\right), \\
u_{j}^{\prime}\left(E_{i}\right) & =\frac{d u_{j}}{d x_{j}}\left(\pi_{j}^{-1}\left(E_{i}\right)\right), \\
u_{j}{ }^{\prime}\left(E_{i}\right) & =\frac{d^{2} u_{j}}{d x_{j}^{2}}\left(\pi_{j}^{-1}\left(E_{i}\right)\right) .
\end{aligned}
$$


Finally, differentiations are carried out on each edge $k_{j}$ with respect to the arc length parameter $x_{j}$.

Let us now fix a $C^{2}$-network $G$ which is a tree (since for such networks the direct and inverse inequalities hold, see below and for instance $[34,24])$. For each edge $k_{j}$, we also fix mechanical constants $m_{j}>0$ (the mass density of the string $k_{j}$ ) and $d_{j}>0$ (the diffusion coefficient of $k_{j}$ ). We now consider the following wave equation:

$$
\left\{\begin{array}{l}
\partial_{t}^{2} u_{j}\left(x_{j}, t\right)-\frac{d_{j}}{m_{j}} u_{j}{ }^{\prime}\left(x_{j}, t\right)=\lambda(t) a_{j}\left(x_{j}\right) \text { in } Q_{j T}, \forall j=1, \cdots, N, \\
u(\cdot, t)=0 \text { is continuous on } G \text { for all } t \in] 0, T[ \\
\left.\sum_{j \in N_{i}} d_{j} \frac{\partial u_{j}}{\partial \nu_{j}}\left(E_{i}, t\right)=0, \forall i \in I_{i n t}, \forall t \in\right] 0, T[ \\
\left.u_{j_{i}}\left(E_{i}, t\right)=0, \forall i \in I_{e x t}, \forall t \in\right] 0, T[ \\
\left.u_{j}\left(x_{j}, 0\right)=\partial_{t} u_{j}\left(x_{j}, 0\right)=0 \text { in }\right] 0, l_{j}[, \forall j=1, \cdots, N,
\end{array}\right.
$$

where $\left.Q_{j T}:=\right] 0, l_{j}[\times] 0, T\left[\right.$ and $\frac{\partial u_{j}}{\partial \nu_{j}}\left(E_{i}, t\right)=\nu_{j}\left(E_{i}\right) u_{j}^{\prime}\left(E_{i}, t\right)$ means the exterior normal derivative of $u_{j}(\cdot, t)$ at $E_{i}$. Above and below $\lambda \in C^{1}([0, T])$ is a given function satisfying

$$
\lambda(0) \neq 0
$$

For all $j=1, \cdots, N$ the datum $a_{j} \in\left(H^{1}\left(0, l_{j}\right)\right)^{\prime}$ is assumed to be in the form

$$
a_{j}\left(x_{j}\right)=\sum_{k=1}^{K_{j}} \alpha_{j k} \delta\left(x_{j}-\xi_{j k}\right),
$$

for some positive integer $K_{j}$, some real numbers $\alpha_{j k}$ different from zero and some (different) points $\xi_{j k}$ in $] 0, l_{j}[$, or more precisely

$$
<a_{j}, \phi>=\sum_{k=1}^{K_{j}} \alpha_{j k} \phi\left(\xi_{j k}\right), \forall \phi \in H^{1}\left(0, l_{j}\right) .
$$

Above and below $H^{p}\left(0, l_{j}\right)$ is the standard Sobolev space of order $p \in \mathbb{N}$ on the interval $10, l_{j}[$.

Our goal is to identify the datum $a$ in the above form (i.e. the location of the point sources $\xi_{j k}$, the weight $\alpha_{j k}$ and the number $K_{j}$ ) from boundary measurements, namely the value of $u_{j_{i}}^{\prime}\left(E_{i}, t\right)$, for $0<t<T$ and all external vertices $E_{i}$ except one.

In order to analyse the system (1) we introduce the following operator $A$ on the Hilbert space $H=\Pi_{j=1}^{N} L^{2}\left(0, l_{j}\right)$, endowed with the inner product

$$
(u, v)_{H}=\sum_{j=1}^{N} m_{j} \int_{0}^{l_{j}} u_{j}(x) v_{j}(x) d x .
$$




$$
\begin{aligned}
& \left\{\begin{array}{r}
D(A)=\left\{u \in H: u_{j} \in H^{2}\left(0, l_{j}\right) \text { satisfying (5) to }(7) \text { hereafter }\right\} \\
\forall u \in D(A): A u=\left(\frac{d_{j}}{m_{j}} u_{j} "\right)_{j=1}^{N} .
\end{array}\right. \\
& u \text { is continuous on } G . \\
& \sum_{j \in N_{i}} d_{j} \frac{\partial u_{j}}{\partial \nu_{j}}\left(E_{i}\right)=0, \forall i \in I_{i n t}, \\
& u_{j_{i}}\left(E_{i}\right)=0, \forall i \in I_{e x t} .
\end{aligned}
$$

Remark that $A$ is a negative selfadjoint operator with a compact resolvant since $A$ is the Friedrichs extension of the triple $(H, V, a)$ defined by

$$
V=\left\{u \in \Pi_{j=1}^{N} H^{1}\left(0, l_{j}\right) \text { satisfying (5) and (7) }\right\},
$$

which is a Hilbert space with the inner product

$$
(u, v)_{V}=\sum_{j=1}^{N} \int_{0}^{l_{j}} u_{j}^{\prime} v_{j}^{\prime} d x_{j},
$$

and

$$
a(u, v)=\sum_{j=1}^{N} d_{j} \int_{0}^{l_{j}} u_{j}^{\prime}\left(x_{j}\right) v_{j}^{\prime}\left(x_{j}\right) d x_{j} .
$$

The spectrum of this operator $A$ was studied in details in $[2,3,4,5,8,7,24,27$, $28,29]$. For our future uses we recall the following Weyl's formula: if $\left\{\lambda_{k}\right\}_{k=1}^{\infty}$ denotes the set of eigenvalues of the operator $-A$ in increasing order and repeated according to their multiplicity, then

$$
\lim _{k \rightarrow \infty} \frac{\lambda_{k}}{k^{2}}=\pi^{2}\left(\sum_{j=1}^{N} \frac{\sqrt{m_{j}} l_{j}}{\sqrt{d_{j}}}\right)^{-2} .
$$

Let us further prove that the eigenvectors are uniformly bounded:

Lemma 2.1. For all positive integer $k$ let $\phi_{k}$ be the eigenvector of $A$ associated with $-\lambda_{k}$. Then denoting by $\phi_{k, j}$ the restriction of $\phi_{k}$ to the edge $j$, for $k$ large enough, we have

$$
\left|\phi_{k, j}\left(x_{j}\right)\right| \leq \frac{2 \sqrt{2} \sqrt{l_{j}}}{\sqrt{m_{j}}}, \forall x_{j} \in\left[0, l_{j}\right] .
$$

Proof. Since $\phi_{k, j}$ satisfies

$$
\left.\frac{d_{j}}{m_{j}} \phi_{k, j} "=-\lambda_{k} \phi_{k, j} \text { on }\right] 0, l_{j}[
$$


there exist real numbers $c_{k, j}$ and $d_{k, j}$ such that

$$
\phi_{k, j}\left(x_{j}\right)=c_{k, j} \cos \left(\sqrt{\frac{m_{j} \lambda_{k}}{d_{j}}} x_{j}\right)+d_{k, j} \sin \left(\sqrt{\frac{m_{j} \lambda_{k}}{d_{j}}} x_{j}\right), \forall x_{j} \in\left[0, l_{j}\right] .
$$

Consequently by integrating the square of this expression in $\left[0, l_{j}\right]$ and writing for shortness $L_{j}=\frac{\sqrt{m_{j}} l_{j}}{\sqrt{d_{j}}}$, we obtain

$$
\begin{aligned}
\sqrt{\frac{m_{j}}{d_{j}}} \int_{0}^{l_{j}}\left|\phi_{k, j}\left(x_{j}\right)\right|^{2} d x_{j} & =c_{k, j}^{2}\left\{\frac{L_{j}}{2}+\frac{\sin \left(2 \sqrt{\lambda_{k}} L_{j}\right)}{4 \sqrt{\lambda_{k}}}\right\}+d_{k, j}^{2}\left\{\frac{L_{j}}{2}-\frac{\sin \left(2 \sqrt{\lambda_{k}} L_{j}\right)}{4 \sqrt{\lambda_{k}}}\right\} \\
& +c_{k, j} d_{k, j}\left\{\frac{1}{2 \sqrt{\lambda_{k}}}-\frac{\cos \left(2 \sqrt{\lambda_{k}} L_{j}\right)}{2 \sqrt{\lambda_{k}}}\right\} .
\end{aligned}
$$

By (9) for $k$ large enough we get

$$
\begin{aligned}
& \frac{L_{j}}{2}+\frac{\sin \left(2 \sqrt{\lambda_{k}} L_{j}\right)}{4 \sqrt{\lambda_{k}}} \geq \frac{L_{j}}{4}, \\
& \frac{L_{j}}{2}-\frac{\sin \left(2 \sqrt{\lambda_{k}} L_{j}\right)}{4 \sqrt{\lambda_{k}}} \geq \frac{L_{j}}{4}, \\
&\left|\frac{1}{2 \sqrt{\lambda_{k}}}-\frac{\cos \left(2 \sqrt{\lambda_{k}} L_{j}\right)}{2 \sqrt{\lambda_{k}}}\right| \leq \frac{L_{j}}{4} .
\end{aligned}
$$

Inserting these estimates in the previous identity we arrive at

$$
\begin{aligned}
\sqrt{\frac{m_{j}}{d_{j}}} \int_{0}^{l_{j}}\left|\phi_{k, j}\left(x_{j}\right)\right|^{2} d x_{j} & \geq\left(c_{k, j}^{2}+d_{k, j}^{2}-\left|c_{k, j} d_{k, j}\right|\right) \frac{L_{j}}{4} \\
& \geq\left(c_{k, j}^{2}+d_{k, j}^{2}\right) \frac{L_{j}}{8}
\end{aligned}
$$

for $k$ large enough. We conclude by noting that

$$
m_{j} \int_{0}^{l_{j}}\left|\phi_{k, j}\left(x_{j}\right)\right|^{2} d x_{j} \leq\left\|\phi_{k, j}\right\|_{H}^{2}=1 .
$$

We are now ready to prove that our wave equation (1) is uniquely solvable and to give regularity of its solution:

Theorem 2.2. The wave equation (1) has a unique (weak) solution u satisfying

$$
u \in C([0, T] ; V) \cap C^{1}([0, T] ; H) .
$$


Proof. We remark that the system (1) is equivalently written

$$
\left\{\begin{array}{l}
\left.\partial_{t}^{2} u=A u+\lambda(t) a \text { in }\right] 0, T[ \\
u(0)=0, \partial_{t} u(0)=0
\end{array}\right.
$$

where $a \in V^{\prime}$ is defined by

$$
<a, \phi>_{V^{\prime}-V}=\sum_{j=1}^{N} m_{j} \sum_{k=1}^{K_{j}} \alpha_{j k} \phi_{j}\left(\xi_{j k}\right), \forall \phi \in V .
$$

The solution of that system is explicitly given by (using spectral expansions)

$$
u(t)=\sum_{k=1}^{\infty} \frac{1}{\sqrt{\lambda_{k}}} \int_{0}^{t} \sin \left((t-s) \sqrt{\lambda_{k}}\right) \lambda(s) d s<a, \phi_{k}>\phi_{k},
$$

or equivalently, by integration by parts in the above integral:

$$
u(t)=\sum_{k=1}^{\infty} \frac{a_{k}(t)}{\lambda_{k}} \phi_{k}
$$

where $a_{k}$ is given by

$$
a_{k}(t)=<a, \phi_{k}>\left(\lambda(t)-\lambda(0) \cos \left(t \sqrt{\lambda_{k}}\right)-\int_{0}^{t} \cos \left((t-s) \sqrt{\lambda_{k}}\right) \lambda^{\prime}(s) d s\right) .
$$

We now remark that Lemma 2.1, the form of $a$ and the smoothness of $\lambda$ allow to conclude the existence of a constant $M$ (depending on $T$ but not on $k$ ) such that

$$
\left|a_{k}(t)\right| \leq M, \forall k=1, \cdots, \infty
$$

By Parseval's identity we have

$$
\|u(t)\|_{V}^{2} \sim\|u(t)\|_{D\left(A^{1 / 2}\right)}^{2} \sim \sum_{k=1}^{\infty} \frac{\left|a_{k}(t)\right|^{2}}{\lambda_{k}}
$$

and consequently by the estimate (14) we conclude that

$$
\|u(t)\|_{V}^{2} \leq M^{2} \sum_{k=1}^{\infty} \frac{1}{\lambda_{k}} \leq C, \forall t \in[0, T],
$$

for some positive constant $C$ (depending on $T$ ) since the asymptotic behaviour of the eigenvalues guarantees the convergence of the series $\sum_{k=1}^{\infty} \frac{1}{\lambda_{k}}$. This means that the series $\sum_{k=1}^{\infty} \frac{a_{k}(t)}{\lambda_{k}} \phi_{k}$ is convergent in $L^{\infty}([0, T] ; V)$ and then proves that

$$
u \in C([0, T] ; V) \text {, }
$$


as limit of elements from $C([0, T] ; V)$ (the truncated series).

Similarly by direct calculations we have

$$
\|\left.\partial_{t} u(t)\right|_{H} ^{2}=\sum_{k=1}^{\infty} \frac{\left|\partial_{t} a_{k}(t)\right|^{2}}{\lambda_{k}^{2}} \leq C \sum_{k=1}^{\infty} \frac{1}{\lambda_{k}}
$$

for some positive constant $C$ (depending on $T$ ), and we conclude as before that $u \in C^{1}([0, T] ; H)$.

\section{Some observability estimates}

In this section we first recall the (standard) direct and inverse inequalities for the wave equation in a tree, obtained in $[34,24]$ for general hyperbolic systems using the multiplier method or the method of characteristics and that easily follows in our case using the multiplier method. Some hidden regularity for our system (1) and some observability estimates for an associated one are secondly deduced. We then consider the wave equation

$$
\left\{\begin{array}{l}
\left.\partial_{t}^{2} \phi-A \phi=f \text { in }\right] 0, T[, \\
u(0)=\phi_{0}, \partial_{t} u(0)=\phi_{1},
\end{array}\right.
$$

where $\left(\phi_{0}, \phi_{1}\right)$ belongs to $V \times H$ and $f \in L^{1}(10, T[; H)$. It is well known that this system has a unique solution $\phi \in C([0, T] ; V) \cap C^{1}([0, T] ; H)$. We now state the so-called identity with multiplier which is the key identity for the direct and inverse inequalities.

Lemma 3.1. Let $T>0$ and let $q: G \rightarrow \mathbb{R}$ be a multiplier with the regularity $q_{j} \in$ $C^{1}\left(\left[0, l_{j}\right]\right)$, for all $j=1, \cdots, N$. Then for all $\left(\phi_{0}, \phi_{1}\right) \in V \times H$ and $f \in L^{1}(] 0, T[; H)$, the solution $\phi \in C([0, T] ; V) \cap C^{1}([0, T] ; H)$ of (15) satisfies

$$
\begin{aligned}
& \frac{1}{2} \sum_{i \in I_{\text {ext }}} \int_{0}^{T} d_{j_{i}}\left|\phi_{j_{i}}^{\prime}\left(E_{i}, t\right)\right|^{2} q_{j_{i}}\left(E_{i}\right) \nu_{j_{i}}\left(E_{i}\right) d t \\
+ & \frac{1}{2} \sum_{i \in I_{i n t}} \sum_{j \in N_{i}} \int_{0}^{T}\left(d_{j}\left|\phi_{j}^{\prime}\left(E_{i}, t\right)\right|^{2}+m_{j}\left|\partial_{t} \phi_{j}\left(E_{i}, t\right)\right|^{2}\right) q_{j}\left(E_{i}\right) \nu_{j}\left(E_{i}\right) d t \\
= & \frac{1}{2} \sum_{j=1}^{N} \int_{Q_{j T}} q_{j}^{\prime}\left(m_{j}\left|\partial_{t} \phi_{j}\right|^{2}+d_{j}\left|\phi_{j}^{\prime}\right|^{2}\right) d x_{j} d t-\sum_{j=1}^{N} m_{j} \int_{Q_{j T}} f_{j} q_{j} \phi_{j}^{\prime} d x_{j} d t \\
+ & \left.\sum_{j=1}^{N} m_{j} \int_{0}^{l_{j}} \partial_{t} \phi_{j} q_{j} \phi_{j}^{\prime} d x_{j}\right|_{0} ^{T} .
\end{aligned}
$$

Proof. This identity is obtained as follows: first multiply the restriction of the first equation of (15) to the edge $j$ by $m_{j} q_{j} \phi_{j}^{\prime}$, secondly apply some integrations by part 
in space and time as in Lemma I.3.7 of [26] for instance and thirdly take the sum on $j$ from 1 to $N$.

This lemma allows to obtain the direct estimate which proves the so-called hidden regularity of $\partial_{n} \phi$ on the external boundary.

Lemma 3.2. Let $T>0$, then there exists a positive constant $c$ such that for all $\left(\phi_{0}, \phi_{1}\right) \in V \times H$ the solution $\phi \in C([0, T] ; V) \cap C^{1}([0, T] ; H)$ of $(15)$ with $f=0$ satisfies

$$
\sum_{i \in I_{e x t}} \int_{0}^{T}\left|u_{j_{i}}^{\prime}\left(E_{i}, t\right)\right|^{2} d t \leq c(T+1) E_{0},
$$

where $E_{0}=E(0)$ is the energy of the system at time $t=0$ and we recall that

$$
E(t)=\frac{1}{2}\left(\left\|\partial_{t} \phi(t)\right\|_{H}^{2}+a(\phi(t), \phi(t))\right)
$$

Proof. In the identity (16) we restrict ourselves to $q$ identically equal to zero near the interior nodes and such that

$$
q_{j_{i}}\left(E_{i}\right) \nu_{j_{i}}\left(E_{i}\right)=1, \forall i \in I_{e x t},
$$

which is always possible. Using the boundedness of $q_{j}$ and $q_{j}^{\prime}$ we obtain

$$
\begin{aligned}
& \frac{1}{2} \sum_{i \in I_{\text {ext }}} \int_{0}^{T} d_{j_{i}}\left|\phi_{j_{i}}^{\prime}\left(E_{i}, t\right)\right|^{2} d t \leq \frac{C_{1}}{2} \sum_{j=1}^{N} \int_{Q_{j} T}\left(m_{j}\left|\partial_{t} \phi_{j}\right|^{2}+d_{j}\left|\phi_{j}^{\prime}\right|^{2}\right) d x_{j} d t(18) \\
+\quad & C_{1} \sum_{j=1}^{N} m_{j} \int_{0}^{l_{j}}\left(\left|\partial_{t} \phi_{j}\left(x_{j}, 0\right) \phi_{j}^{\prime}\left(x_{j}, 0\right)\right|+\left|\partial_{t} \phi_{j}\left(x_{j}, T\right) \phi_{j}^{\prime}\left(x_{j}, T\right)\right|\right) d x_{j},
\end{aligned}
$$

for some positive constant $C_{1}$ (independent of $T$ ). By the conservation of energy we have

$$
\frac{1}{2} \sum_{j=1}^{N} \int_{Q_{j T}}\left(m_{j}\left|\partial_{t} \phi_{j}\right|^{2}+d_{j}\left|\phi_{j}^{\prime}\right|^{2}\right) d x_{j} d t=T E_{0}
$$

On the other hand by Cauchy-Schwarz's inequality, for $t \in[0, T]$ we may estimate

$$
\sum_{j=1}^{N} m_{j} \int_{0}^{l_{j}}\left|\partial_{t} \phi_{j}\left(x_{j}, t\right) \phi_{j}^{\prime}\left(x_{j}, t\right)\right| d x_{j} \leq C_{2} E(t)=C_{2} E(0),
$$

for some positive constant $C_{2}$ (independent of $T$ ). Consequently we have

$$
\sum_{j=1}^{N} m_{j} \int_{0}^{l_{j}}\left(\left|\partial_{t} \phi_{j}\left(x_{j}, 0\right) \phi_{j}^{\prime}\left(x_{j}, 0\right)\right|+\left(\left|\partial_{t} \phi_{j}\left(x_{j}, T\right) \phi_{j}^{\prime}\left(x_{j}, T\right)\right|\right) d x_{j} \leq 2 C_{2} E_{0} .\right.
$$


The above identity and the estimate (19) in (18) yield the conclusion.

Let us now pass to the inverse estimate.

Lemma 3.3. Let $T>0$ and fix one exterior vertex $E_{i_{0}}$ of $G$ (called the root of $G$ ). Then there exist a positive constant $c^{\prime}$ and a positive time $T_{0}$ which depend only on $l_{j}, m_{j}, d_{j}$ and on the algebraic structure of $G$ such that for all $\left(\phi_{0}, \phi_{1}\right) \in V \times H$ the solution $\phi \in C([0, T] ; V) \cap C^{1}([0, T] ; H)$ of (15) with $f=0$ satisfies

$$
c^{\prime}\left(T-T_{0}\right) E_{0} \leq \sum_{i \in I_{\text {ext }} \backslash\left\{i_{0}\right\}} \int_{0}^{T}\left|u_{j_{i}}^{\prime}\left(E_{i}, t\right)\right|^{2} d t .
$$

Proof. In the identity (16) we restrict ourselves to a multiplier $q$ such that in the left-hand side of (16) the contribution of the interior nodes is nonpositive and the contribution of the node $E_{i_{0}}$ is zero. So we look for $q$ satisfying $q_{j_{i_{0}}}\left(E_{i_{0}}\right)=0$ and for all $i \in I_{\text {int }}$ :

$$
\begin{aligned}
& \sum_{j \in N_{i}} m_{j} q_{j}\left(E_{i}\right) \nu_{j}\left(E_{i}\right) \leq 0, \\
& \sum_{j \in N_{i}} d_{j} q_{j}\left(E_{i}\right) \nu_{j}\left(E_{i}\right)\left|\beta_{j}\right|^{2} \leq 0,
\end{aligned}
$$

for all $\left(\beta_{j}\right)_{j \in N_{i}}$ such that

$$
\sum_{j \in N_{i}} d_{j} \nu_{j}\left(E_{i}\right) \beta_{j}=0 .
$$

The first condition comes from the fact that

$$
\sum_{j \in N_{i}} \int_{0}^{T} m_{j}\left|\partial_{t} \phi_{j}\left(E_{i}, t\right)\right|^{2} q_{j}\left(E_{i}\right) \nu_{j}\left(E_{i}\right) d t=\int_{0}^{T}\left|\partial_{t} \phi\left(E_{i}, t\right)\right|^{2} d t \sum_{j \in N_{i}} m_{j} q_{j}\left(E_{i}\right) \nu_{j}\left(E_{i}\right)
$$

since $\phi(\cdot, t)$ is continuous at $E_{i}$. The second condition comes from the fact that

$$
\sum_{j \in N_{i}} \int_{0}^{T} d_{j}\left|\phi_{j}^{\prime}\left(E_{i}, t\right)\right|^{2} q_{j}\left(E_{i}\right) \nu_{j}\left(E_{i}\right) d t=\int_{0}^{T} \sum_{j \in N_{i}} d_{j} q_{j}\left(E_{i}\right) \nu_{j}\left(E_{i}\right)\left|\phi_{j}^{\prime}\left(E_{i}, t\right)\right|^{2} d t,
$$

and recalling that $\phi$ satisfies (6).

To build such a $q$ we classify the edges of $G$ into generations: The first generation is $j_{i_{0}}$, the second generation is the edges (different from $j_{i_{0}}$ ) which have a node in common with $j_{i_{0}}$, and by iteration the $(i+1)^{t h}$ generation is the edges which do not belong to the $i^{t h}$ generation and have a node in common with an edge of the $i^{\text {th }}$ generation. On each edge $j$ of the $i^{\text {th }}$ generation with $i \geq 1$, we now use the parametrization $\pi_{j}$ such that $\pi_{j}(0)$ is a node belonging to the $(i-1)^{t h}$ generation if $i \geq 2$ and $\pi_{j}(0)=E_{i_{0}}$ if $i=1$. 
With these notation we first take

$$
q_{j_{i_{0}}}(x)=x,
$$

which is nonnegative and clearly satisfies $q_{j_{0}}\left(E_{i_{0}}\right)=0$. We now build $q \geq 0$ iteratively (from generation to generation) in order to satisfy the conditions (21) and (22). But we remark that these conditions are equivalent to the following ones: for all node $E_{i}$ between an edge $k$ of the $i^{t h}$ generation (which is unique) and the $(i+1)^{t h}$ generation, we require

$$
\begin{aligned}
& m_{k} q_{k}\left(E_{i}\right) \leq \sum_{j \in N_{i}, j \neq k} m_{j} q_{j}\left(E_{i}\right) \\
& d_{k} q_{k}\left(E_{i}\right)\left|\beta_{k}\right|^{2}-\sum_{j \in N_{i}, j \neq k} d_{j} q_{j}\left(E_{i}\right)\left|\beta_{j}\right|^{2} \leq 0,
\end{aligned}
$$

for all $\left(\beta_{j}\right)_{j \in N_{i}}$ such that

$$
d_{k} \beta_{k}=\sum_{j \in N_{i}, j \neq k} d_{j} \beta_{j}
$$

This last condition (24) may be then equivalently written as

$$
d_{k} q_{k}\left(E_{i}\right)\left(\sum_{j \in N_{i}, j \neq k} \frac{d_{j}}{d_{k}} \beta_{j}\right)^{2}-\sum_{j \in N_{i}, j \neq k} d_{j} q_{j}\left(E_{i}\right)\left|\beta_{j}\right|^{2} \leq 0
$$

for all $\left(\beta_{j}\right)_{j \in N_{i} \backslash\{k\}}$. To eliminate these parameters $\beta_{j}$, we use the estimate

$$
\left(\sum_{j \in N_{i}, j \neq k} \frac{d_{j}}{d_{k}} \beta_{j}\right)^{2} \leq C \sum_{j \in N_{i}, j \neq k} \frac{d_{j}^{2}}{d_{k}^{2}} \beta_{j}^{2},
$$

which holds for a positive constant $C$ which normally depends on the cardinal of the set $N_{i} \backslash\{k\}$ and that we then estimate from above by a constant independent of that cardinal and which depends on the number of edges of $G$.

Therefore (25) holds if we have

$$
C q_{k}\left(E_{i}\right) \frac{d_{j}^{2}}{d_{k}} \leq d_{j} q_{j}\left(E_{i}\right), \forall j \in N_{i} \backslash\{k\},
$$

or equivalently

$$
C q_{k}\left(E_{i}\right) \frac{d_{j}}{d_{k}} \leq q_{j}\left(E_{i}\right), \forall j \in N_{i} \backslash\{k\} .
$$

In summary we are looking for $q$ satisfying (23) and (26) at all nodes $E_{i}$ between an edge $k$ of the $i^{t h}$ generation and the $(i+1)^{t h}$ generation. For that purpose we take

$$
q_{j}(x)=\alpha(x+1), \forall j \in N_{i} \backslash\{k\},
$$


for some $\alpha>0$ which depends on $E_{i}$ and that will be fixed below. With this choice we see that (since $\left.q_{j}\left(E_{i}\right)=\alpha\right)(23)$ and (26) are respectively equivalent to

$$
\begin{aligned}
& \frac{m_{k}}{\sum_{j \in N_{i}, j \neq k} m_{j}} q_{k}\left(E_{i}\right) \leq \alpha, \\
& C q_{k}\left(E_{i}\right) \frac{d_{j}}{d_{k}} \leq \alpha .
\end{aligned}
$$

Since we assume that $q_{k}$ is known (by inductive hypothesis) we see that the two above conditions yield a finite number of lower bounds to $\alpha$ and therefore such a $\alpha$ always exists.

By induction we have built $q$.

Taking such a $q$ in the identity with multiplier we have

$$
\begin{aligned}
& \frac{1}{2} \sum_{j=1}^{N} \int_{Q_{j} T} q_{j}^{\prime}\left(m_{j}\left|\partial_{t} \phi_{j}\right|^{2}+d_{j}\left|\phi_{j}^{\prime}\right|^{2}\right) d x_{j} d t+\left.\sum_{j=1}^{N} m_{j} \int_{0}^{l_{j}} \partial_{t} \phi_{j} q_{j} \phi_{j}^{\prime} d x_{j}\right|_{0} ^{T} \\
& \leq \frac{1}{2} \sum_{i \in I_{\text {ext }} \backslash\left\{i_{0}\right\}} \int_{0}^{T} d_{j_{i}}\left|\phi_{j_{i}}^{\prime}\left(E_{i}, t\right)\right|^{2} q_{j_{i}}\left(E_{i}\right) \nu_{j_{i}}\left(E_{i}\right) d t
\end{aligned}
$$

We conclude by the estimate (19) and the fact that $q_{j}^{\prime}$ are uniformly bounded from below.

Using the direct and inverse estimates and the arguments of Theorem I.6.3 of [26], we obtain the next (weak) observability estimates:

Lemma 3.4. For $a \in V^{\prime}$ there exists a unique solution $v \in C([0, T] ; H) \cap C^{1}\left([0, T] ; V^{\prime}\right)$ of

$$
\left\{\begin{array}{l}
\left.\partial_{t}^{2} v-A v=0 \text { in }\right] 0, T[ \\
v(0)=0, \partial_{t} v(0)=a
\end{array}\right.
$$

Moreover for $T>T_{0}$ with $T_{0}$ from Lemma 3.3, there exist two positive constants $C_{1}$ and $C_{2}$ depending on $T$ such that

$$
C_{1}\|a\|_{V^{\prime}} \leq \sum_{i \in I_{e x t} \backslash\left\{i_{0}\right\}}\left\|v_{j_{i}}^{\prime}\left(E_{i}, \cdot\right)\right\|_{H^{-1}(0, T)} \leq C_{2}\|a\|_{V^{\prime}},
$$

where, as usual, $H^{-1}(0, T)$ is the dual space of $H_{0}^{1}(0, T)$.

Let us also give a consequence of the identity with multiplier to the solution $u$ of problem (1), namely the hidden regularity of $\partial_{n} u$ on the external boundary:

Lemma 3.5. Let $u \in C([0, T] ; V) \cap C^{1}([0, T] ; H)$ be the unique solution of (1) with datum $a_{j}$ in the form (3) or more precisely solution of (11) with datum a in the form 
(12). Then for all $T>0$ and all $i \in I_{e x t}, u_{j_{i}}^{\prime}\left(E_{i}, \cdot\right)$ belongs to $L^{2}(0, T)$ with the estimate

$$
\sum_{i \in I_{e x t}}\left\|u_{j_{i}}^{\prime}\left(E_{i}, \cdot\right)\right\|_{L^{2}(0, T)} \leq C\left(\|u\|_{C([0, T] ; V)}+\|u\|_{C^{1}([0, T] ; H)}\right),
$$

for some positive constant $C$ depending on $T$ and $a_{j}$.

Proof. We approximate $a$ by a sequence of $a_{n} \in V$ such that

$$
a_{n} \rightarrow a \in V^{\prime} \text { as } n \rightarrow \infty \text {. }
$$

Namely for $n$ large enough, we take $a_{n}$ in the form

$$
a_{n}=\sum_{j=1}^{N} \sum_{k=1}^{K_{j}} \alpha_{j k} \phi_{j k n},
$$

where

$$
\phi_{j k n}(x)=n \phi\left(n\left(x-\xi_{j k}\right)\right), \forall x \in\left[0, l_{j}\right],
$$

with a fixed nonnegative function $\phi \in \mathcal{D}(\mathbb{R})$ with a support in $[-1,1]$ and such that $\int_{-1}^{1} \phi(x) d x=1$. The above convergence property follows from the easily checked property:

$$
\left|\int_{\mathbb{R}} n \phi\left(n\left(x-\xi_{j k}\right)\right) \chi(x) d x-\chi\left(\xi_{j k}\right)\right| \leq \frac{1}{\sqrt{n}}\left\|\chi^{\prime}\right\|_{L^{2}(0,1)},
$$

valid for $n$ large enough.

We further remark that for all eigenvectors $\phi_{k^{\prime}}$, we may write

$$
\begin{aligned}
<a_{n}-a, \phi_{k^{\prime}}> & =\left(a_{n}, \phi_{k^{\prime}}\right)_{H}-<a, \phi_{k^{\prime}}> \\
& =\sum_{j=1}^{N} m_{j} \sum_{k=1}^{K_{j}} \alpha_{j k}\left(\int_{0}^{l_{j}} \phi_{j k n}(x) \phi_{k^{\prime} j}(x) d x-\phi_{k^{\prime} j}\left(\xi_{j k}\right)\right) \\
& =\sum_{j=1}^{N} m_{j} \sum_{k=1}^{K_{j}} \alpha_{j k} \int_{-1}^{1} \phi(y)\left(\phi_{k^{\prime} j}\left(\xi_{j k}+\frac{y}{n}\right)-\phi_{k^{\prime} j}\left(\xi_{j k}\right)\right) d y .
\end{aligned}
$$

Therefore by Lemma 2.1 we conclude that there exists a positive constant $M$ (independent of $k^{\prime}$ ) such that

$$
\left|<a_{n}-a, \phi_{k^{\prime}}>\right| \leq M, \forall k^{\prime}=1, \cdots, \infty .
$$

Let $u_{n}$ be the solution of (11) with datum $a_{n}$, which, by spectral expansions, satisfies

$$
u_{n} \in C^{2}([0, T] ; H) \cap C^{1}([0, T] ; V) \cap C([0, T] ; D(A)) .
$$


Furthermore by the property (31), the estimate (32) and Lebesgue's bounded convergence Theorem, we have (see Theorem 2.2)

$$
u_{n} \rightarrow u \text { in } C([0, T] ; V) \cap C^{1}([0, T] ; H) \text { as } n \rightarrow \infty .
$$

Due to the regularity (33) we can apply the identity with multiplier (16) from Lemma 3.1 to $u_{n}$ to get

$$
\begin{aligned}
& \frac{1}{2} \sum_{i \in I_{\text {ext }}} \int_{0}^{T} d_{j_{i}}\left|u_{n j_{i}}^{\prime}\left(E_{i}, t\right)\right|^{2} q_{j_{i}}\left(E_{i}\right) \nu_{j_{i}}\left(E_{i}\right) d t \\
+ & \frac{1}{2} \sum_{i \in I_{\text {int }}} \sum_{j \in N_{i}} \int_{0}^{T}\left(d_{j}\left|\phi_{j}^{\prime}\left(E_{i}, t\right)\right|^{2}+m_{j}\left|\partial_{t} u_{n j}\left(E_{i}, t\right)\right|^{2}\right) q_{j}\left(E_{i}\right) \nu_{j}\left(E_{i}\right) d t \\
= & \frac{1}{2} \sum_{j=1}^{N} \int_{Q_{j} T} q_{j}^{\prime}\left(m_{j}\left|\partial_{t} u_{n j}\right|^{2}+d_{j}\left|u_{n j}^{\prime}\right|^{2}\right) d x_{j} d t-\sum_{j=1}^{N} m_{j} \int_{Q_{j T}} \lambda(t) a_{n j} q_{j} u_{n j}^{\prime} d x_{j} d t \\
+ & \left.\sum_{j=1}^{N} m_{j} \int_{0}^{l_{j}} \partial_{t} u_{n j} q_{j} u_{n j}^{\prime} d x_{j}\right|_{0} ^{T} .
\end{aligned}
$$

Choosing $q_{j}$ identically equal to zero in a neighbourhood of the points $\xi_{j k}$ for all $j, k$, identically equal to zero in a neighbourhood of the interior vertices and such that

$$
q_{j_{i}}\left(E_{i}\right) \nu_{j_{i}}\left(E_{i}\right)=\frac{1}{d_{j_{i}}}, \forall i \in I_{e x t},
$$

the above identity becomes

$$
\begin{aligned}
& \frac{1}{2} \sum_{i \in I_{\text {ext }}} \int_{0}^{T}\left|u_{n j_{i}}^{\prime}\left(E_{i}, t\right)\right|^{2} d t \\
= & \frac{1}{2} \sum_{j=1}^{N} \int_{Q_{j T}} q_{j}^{\prime}\left(m_{j}\left|\partial_{t} u_{n j}\right|^{2}+d_{j}\left|u_{n j}^{\prime}\right|^{2}\right) d x_{j} d t \\
+ & \left.\sum_{j=1}^{N} m_{j} \int_{0}^{l_{j}} \partial_{t} u_{n j} q_{j} u_{n j}^{\prime} d x_{j}\right|_{0} ^{T},
\end{aligned}
$$

for $n$ large enough. By Cauchy-Schwarz's inequality we arrive at

$$
\sum_{i \in I_{e x t}} \int_{0}^{T}\left|u_{n j_{i}}^{\prime}\left(E_{i}, t\right)\right|^{2} d t \leq C\left(\left\|u_{n}\right\|_{C([0, T] ; V)}^{2}+\left\|u_{n}\right\|_{C^{1}([0, T] ; H)}^{2}\right),
$$

for some positive constant $C$ depending on $T$ and $q_{j}$ (and then on $a_{j}$ ).

We conclude by passing to the limit in $n$ owing to the property (34) and Theorem 2.2 . 


\section{Uniqueness}

We first recall Duhamel's principle (see for instance [36, 10]) which gives the relationship between $v$ solution of (28) and $u$ solution of (1).

Lemma 4.1. Let $u \in C([0, T] ; V) \cap C^{1}([0, T] ; H)$ be the unique solution of (1) with datum $a_{j}$ in the form (3) or more precisely solution of (11) with datum a in the form (12) and let $v \in C([0, T] ; H) \cap C^{1}\left([0, T] ; V^{\prime}\right)$ be the unique solution of (28) with initial speed $a$. Then

$$
u(t)=(K v)(t), \forall t \in] 0, T[
$$

where $K$ is defined by

$$
\left.(K \psi)(t)=\int_{0}^{t} \lambda(t-s) \psi(s) d s, \forall t \in\right] 0, T[,
$$

and is a bounded operator from $L^{2}(0, T)$ into itself.

Proof. As in Lemma 3.5 we approximate $a$ by a sequence of $a_{n} \in V$ satisfying (31) and consider the solution $u_{n}$ of (11) with datum $a_{n}$ satisfying (33) and (34).

Similarly the unique solution $v_{n}$ of $(28)$ with initial speed $a_{n}$ satisfies

$$
v_{n} \in C^{2}([0, T] ; H) \cap C^{1}([0, T] ; V) \cap C([0, T] ; D(A)),
$$

and by Lemma 3.4

$$
v_{n} \rightarrow v \text { in } C([0, T] ; H) \cap C^{1}\left([0, T] ; V^{\prime}\right) \text { as } n \rightarrow \infty .
$$

For $u_{n}$ and $v_{n}$, we clearly have

$$
\left.u_{n}(t)=\left(K v_{n}\right)(t), \forall t \in\right] 0, T[,
$$

since we simply check that the right-hand side of this identity satisfies the same problem than the left-hand side.

We conclude by passing to the limit in (37) using the continuity of $K$ from $L^{2}(0, T)$ into itself and the above convergence of $u_{n}$ (resp. $v_{n}$ ) to $u$ (resp. $v$ ).

For further uses, as in [10] we need to extend the above operator $K$ to the space $H_{-1}(0, T)$ defined as the dual space of

$$
{ }^{0} H^{1}(0, T)=\left\{v \in H^{1}(0, T): v(T)=0\right\},
$$

which is a Hilbert space with the norm

$$
\|v\|_{{ }^{0} H^{1}(0, T)}=\left(\int_{0}^{T}\left|\partial_{t} v(t)\right|^{2} d t\right)^{1 / 2} .
$$


As the identity mapping from $H_{0}^{1}(0, T)$ to ${ }^{0} H^{1}(0, T)$ is clearly continuous, its adjoint operator $I d^{\star}$ from $H_{-1}(0, T)$ into $H^{-1}(0, T)$ is continous (but not injective). In other words, for $h \in H_{-1}(0, T)$, we have

$$
<I d^{\star} h, \phi>_{H^{-1}(0, T)-H_{0}^{1}(0, T)}=<h, \phi>_{H_{-1}(0, T)-{ }^{0} H^{1}(0, T)}, \forall \phi \in H_{0}^{1}(0, T),
$$

and moreover $\left\|I d^{\star} h\right\|_{H^{-1}(0, T)} \leq\|h\|_{H_{-1}(0, T)}$. Note that $I d^{\star} h$ is simply the restriction of $h$ to $H_{0}^{1}(0, T)$.

We can now recall the following result (mainly) proved in [10]:

Lemma 4.2. If $\lambda \in C^{1}([0, T])$ satisfies (2) then the bounded operator $K$ from $L^{2}(0, T)$ into itself defined by (36) can be extended to a bounded operator from $H_{-1}(0, T)$ onto $L^{2}(0, T)$ and satisfying

$$
C_{1}\|K \psi\|_{L^{2}(0, T)} \leq C_{1}\|\psi\|_{H_{-1}(0, T)} \leq C_{2}\|K \psi\|_{L^{2}(0, T)}, \forall \psi \in H_{-1}(0, T),
$$

for some positive constants $C_{1}, C_{2}$.

Proof. The extension property of $K$ and the estimates in (38) are proved in Lemma 2 of [10] and are based on the identity

$$
\left(K \psi, \xi^{\prime}\right)_{L^{2}(0, T)}=-(\psi, F \xi)_{L^{2}(0, T)},
$$

valid for all $\psi \in L^{2}(0, T)$ and all $\xi \in{ }^{0} H^{1}(0, T)$, where the operator $F$ is defined by

$$
(F \xi)(t)=\lambda(0) \xi(t)+\int_{t}^{T} \lambda^{\prime}(s-t) \xi(s) d s, 0<t<T,
$$

and is an isomorphism from ${ }^{0} H^{1}(0, T)$ onto itself.

The surjectivity property of $K$ comes from the fact that the assumption (2) guarantees that the range of $K$ as operator from $L^{2}(0, T)$ into itself is equal to (owing to the identity (6.3) of [36] and the change of variable $\tilde{t}=T-t$, see also section 7 of [11])

$$
\left\{v \in H^{1}(0, T): v(0)=0\right\} .
$$

Therefore the range of its extension is dense in $L^{2}(0, T)$ and then equal to $L^{2}(0, T)$ by its closeness, consequence of the estimate (38).

Since the estimate (29) from Lemma 3.4 is only valid for the $H^{-1}(0, T)$-norm of $v_{j_{i}}^{\prime}\left(E_{i}, \cdot\right)$ we actually need to adapt the above Lemma to the space $H^{-1}(0, T)$. For that purpose we need to introduce the subspace of $L^{2}(0, T)$ :

$$
\Lambda^{\perp}=\left\{\eta \in L^{2}(0, T):(\lambda, \eta)_{L^{2}(0, T)}=0\right\},
$$

which means that $\Lambda^{\perp}$ is the closed subspace of $L^{2}(0, T)$ made of functions perpendicular to $\lambda$. We denote by $P$ the orthogonal projection (in $\left.L^{2}(0, T)\right)$ on $\Lambda^{\perp}$. Now we can state the 
Lemma 4.3. If $\lambda \in C^{1}([0, T])$ satisfies (2) then the bounded operator $P K$ from $L^{2}(0, T)$ into itself can be extended to a bounded operator from $H^{-1}(0, T)$ into $L^{2}(0, T)$ and satisfying

$$
C_{3}\|P K \psi\|_{L^{2}(0, T)} \leq\|\psi\|_{H^{-1}(0, T)} \leq C_{4}\|P K \psi\|_{L^{2}(0, T)}, \forall \psi \in H^{-1}(0, T),
$$

for some positive constants $C_{3}, C_{4}$.

Proof. Since $H_{0}^{1}(0, T)$ is a closed subspace of ${ }^{0} H^{1}(0, T)$, the restriction of the operator $F$ to $W=F^{-1}\left(H_{0}^{1}(0, T)\right)$ is an isomorphism from $W$ into $H_{0}^{1}(0, T)$. By the characterization of $H_{0}^{1}(0, T)$ we clearly have

$$
W=\left\{\xi \in{ }^{0} H^{1}(0, T):(F \xi)(0)=0\right\} .
$$

Thanks to the identity

$$
(F \xi)(0)=\lambda(0) \xi(0)+\int_{0}^{T} \lambda^{\prime}(s) \xi(s) d s=-\int_{0}^{T} \lambda(s) \xi^{\prime}(s) d s, \forall \xi \in{ }^{0} H^{1}(0, T),
$$

we equivalently have

$$
W=\left\{\xi \in{ }^{0} H^{1}(0, T): \int_{0}^{T} \lambda(s) \xi^{\prime}(s) d s=0\right\} .
$$

Now we introduce the differentiation operator

$$
D:{ }^{0} H^{1}(0, T) \rightarrow L^{2}(0, T): \xi \rightarrow \xi^{\prime},
$$

which is an isomorphism since for any $\eta \in L^{2}(0, T)$, the function $\xi$ given by

$$
\xi(t)=-\int_{t}^{T} \eta(s) d s
$$

clearly belongs to ${ }^{0} H^{1}(0, T)$ and satisfies $D \xi=\eta$.

Therefore $W$ can be characterized by

$$
W=\left\{\xi \in{ }^{0} H^{1}(0, T): D \xi \in \Lambda^{\perp}\right\} .
$$

Let us now come back to our extension property: Fix $\xi \in L^{2}(0, T)$, then by the usual embedding of $L^{2}(0, T)$ into $H^{-1}(0, T)$ we may write

$$
\|\psi\|_{H^{-1}(0, T)}=\sup _{\phi \in H_{0}^{1}(0, T), \phi \neq 0} \frac{\left|(\psi, \phi)_{L^{2}(0, T)}\right|}{\left\|\phi^{\prime}\right\|_{L^{2}(0, T)}} .
$$

As $F$ is an isomorphism from $W$ into $H_{0}^{1}(0, T)$ we then have

$$
\|\psi\|_{H^{-1}(0, T)} \sim \sup _{\xi \in W, \xi \neq 0} \frac{\left|(\psi, F \xi)_{L^{2}(0, T)}\right|}{\left\|\xi^{\prime}\right\|_{L^{2}(0, T)}} .
$$


By the identity (39) we obtain

$$
\|\psi\|_{H^{-1}(0, T)} \sim \sup _{\xi \in W, \xi \neq 0} \frac{\left|\left(K \psi, \xi^{\prime}\right)_{L^{2}(0, T)}\right|}{\left\|\xi^{\prime}\right\|_{L^{2}(0, T)}} .
$$

By the characterization (41) and the isomorphic property of $D$, we arrive at

$$
\|\psi\|_{H^{-1}(0, T)} \sim \sup _{\eta \in \Lambda^{\perp}, \eta \neq 0} \frac{\left|(K \psi, \eta)_{L^{2}(0, T)}\right|}{\|\eta\|_{L^{2}(0, T)}} .
$$

We conclude by the density of $L^{2}(0, T)$ into $H^{-1}(0, T)$.

Corollary 4.4. Let $u \in C([0, T] ; V) \cap C^{1}([0, T] ; H)$ be the unique solution of (11) with datum $a$ in the form (12) and let $v \in C([0, T] ; H) \cap C^{1}\left([0, T] ; V^{\prime}\right)$ be the unique solution of (28) with initial speed $a$. Then for all $T>0$ and all $i \in I_{\text {ext }}$ we have

$$
P u_{j_{i}}^{\prime}\left(E_{i}, \cdot\right)=P K v_{j_{i}}^{\prime}\left(E_{i}, \cdot\right) \text { in } L^{2}(0, T) \text {. }
$$

Proof. As in Lemma 4.1 let $u_{n}$ (resp. $v_{n}$ ) be the solution of (11) (resp. (28)) with datum $a_{n}$ (resp. with initial speed $a_{n}$ ) with $a_{n} \in V$ satisfying (31).

For these solutions their regularity and Lemma 4.1 allow to write

$$
u_{n j_{i}}^{\prime}\left(E_{i}, \cdot\right)=K v_{n j_{i}}^{\prime}\left(E_{i}, \cdot\right) \text { in } L^{2}(0, T) .
$$

And therefore

$$
P u_{n j_{i}}^{\prime}\left(E_{i}, \cdot\right)=P K v_{n j_{i}}^{\prime}\left(E_{i}, \cdot\right) \text { in } L^{2}(0, T) .
$$

We conclude by passing to the limit in $n$ and using Lemmas 4.3, 3.4 and 3.5.

We are now ready to formulate the uniqueness result:

Theorem 4.5. Let $u^{1}$ (resp. $\left.u^{2}\right)$ in $C([0, T] ; V) \cap C^{1}([0, T] ; H)$ be the unique solution of (11) with datum $a^{1}$ (resp. $\left.a^{2}\right)$ in the form

$$
<a^{l}, \phi>_{V^{\prime}-V}=\sum_{j=1}^{N} m_{j} \sum_{k=1}^{K_{j}^{l}} \alpha_{j k}^{l} \phi_{j}\left(\xi_{j k}^{l}\right), \forall \phi \in V, l=1,2,
$$

for some positive integers $K_{j}^{l}$, real numbers $\alpha_{j k}^{l}$ and points $\left.\xi_{j k}^{l} \in\right] 0, l_{j}[$. Fix one exterior vertex $E_{i_{0}}$ of $G$ and $T>T_{0}$ with $T_{0}>0$ from Lemma 3.3. If for all $i \in$ $I_{\text {ext }} \backslash\left\{i_{0}\right\}$

$$
\left(u^{1}\right)_{j_{i}}^{\prime}\left(E_{i}, t\right)=\left(u^{2}\right)_{j_{i}}^{\prime}\left(E_{i}, t\right), \forall t \in(0, T),
$$

as elements of $L^{2}(0, T)$, then $a^{1}=a^{2}$, or equivalently $K_{j}^{1}=K_{j}^{2}, \alpha_{j k}^{1}=\alpha_{j k}^{2}$ and $\xi_{j k}^{1}=\xi_{j k}^{2}$. 
Proof. We remark that $u=u^{1}-u^{2}$ satisfies (11) with datum $a=a^{1}-a^{2}$ which is still in the form (12). By the assumption we further have

$$
u_{j_{i}}^{\prime}\left(E_{i}, \cdot\right)=0 \text { in } L^{2}(0, T), \forall i \in I_{e x t} \backslash\left\{i_{0}\right\} .
$$

This implies that

$$
P u_{j_{i}}^{\prime}\left(E_{i}, \cdot\right)=0 \text { in } L^{2}(0, T), \forall i \in I_{e x t} \backslash\left\{i_{0}\right\} .
$$

Therefore by Corollary 4.4 and Lemma 4.3 we get

$$
v_{j_{i}}^{\prime}\left(E_{i}, \cdot\right)=0 \text { in } H^{-1}(0, T), \forall i \in I_{e x t} \backslash\left\{i_{0}\right\},
$$

where $v$ is the unique solution of (28) with initial speed $a$. The application of Lemma 3.4 allows to conclude that $a=0$.

\section{Stability}

For fixed positive integers $K_{j}, j=1, \cdots, N$, we denote by

$$
\Sigma=\left\{A=\left(\left(\alpha_{j k}, \xi_{j k}\right)_{k=1}^{K_{j}}\right)_{j=1}^{N}: \alpha_{j k} \in \mathbb{R} \backslash\{0\}, \xi_{j k} \in\right] 0, l_{j}[\} .
$$

The above uniqueness result implies that the mapping

$$
\eta: \Sigma \rightarrow\left(L^{2}(0, T)\right)^{M}: A:=\left(\left(\alpha_{j k}, \xi_{j k}\right)_{k=1}^{K_{j}}\right)_{j=1}^{N} \rightarrow u^{\prime}:=\left(u_{j_{i}}^{\prime}\left(E_{i}, t\right)\right)_{i \in I_{e x t} \backslash\left\{i_{0}\right\}},
$$

where $u$ is the unique solution of (11) with datum $a$ in the form (12), is injective. The stability means that the inverse mapping $\eta^{-1}: u^{\prime} \rightarrow A$ is continuous once $\Sigma$ is equipped with the natural distance

$$
d\left(A^{1}, A^{2}\right)=\sum_{j=1}^{N} \sum_{k=1}^{K_{j}}\left(\left|\alpha_{j k}^{1}-\alpha_{j k}^{2}\right|+\left|\xi_{j k}^{1}-\xi_{j k}^{2}\right|\right),
$$

when

$$
A^{l}:=\left(\left(\alpha_{j k}^{l}, \xi_{j k}^{l}\right)_{k=1}^{K_{j}}\right)_{j=1}^{N}, l=1,2 .
$$

We actually will show a slightly weaker result than the continuity of this mapping by only showing that the inverse of the restriction of $\eta$ to the ball $B(A, \epsilon)$ is locally Lipschitz for some $\epsilon>0$ small enough depending on $A$. Namely we take

$$
\begin{aligned}
\epsilon & \leq \frac{1}{2} \min _{j, k \neq k^{\prime}}\left|\xi_{j k}-\xi_{j k^{\prime}}\right|, \\
\epsilon & \leq \frac{1}{2} \min _{j, k}\left|\xi_{j k}\right|, \\
\epsilon & \leq \frac{1}{2} \min _{j, k}\left|\xi_{j k}-l_{j}\right|, \\
\epsilon & \leq \frac{1}{2} \min _{j, k}\left|\alpha_{j k}\right| .
\end{aligned}
$$


Under these assumptions we can prove the following conditional stability result:

Theorem 5.1. Fix one exterior vertex $E_{i_{0}}$ of $G$ and $T>T_{0}$ with $T_{0}>0$ from Lemma 3.3. Suppose that $A^{2}=\left(\left(\alpha_{j k}^{2}, \xi_{j k}^{2}\right)_{k=1}^{K_{j}}\right)_{j=1}^{N}$ is in $\Sigma \cap B(A, \epsilon)$ with $\epsilon>0$ satisfying the above constraints. Then there exists a constant $C$ depending on $T$, $\min _{j, k \neq k^{\prime}}\left|\xi_{j k}-\xi_{j k^{\prime}}\right|$ and $\min _{j, k}\left|\alpha_{j k}\right|$ such that

$\sum_{j=1}^{N} \sum_{k=1}^{K_{j}}\left(\left|\alpha_{j k}-\alpha_{j k}^{2}\right|+\left|\xi_{j k}-\xi_{j k}^{2}\right|\right) \leq C(1+\sqrt{\epsilon}) \sum_{i \in I_{e x t} \backslash\left\{i_{0}\right\}}\left\|u_{j_{i}}^{\prime}\left(E_{i}, t\right)-\left(u^{2}\right)_{j_{i}}^{\prime}\left(E_{i}, t\right)\right\|_{L^{2}(0, T)}$.

Proof. The proof of Theorem 4.5 clearly shows that

$$
\left\|a-a^{2}\right\|_{V^{\prime}} \leq C \sum_{i \in I_{e x t} \backslash\left\{i_{0}\right\}}\left\|u_{j_{i}}^{\prime}\left(E_{i}, t\right)-\left(u^{2}\right)_{j_{i}}^{\prime}\left(E_{i}, t\right)\right\|_{L^{2}(0, T)} .
$$

Therefore it remains to estimate from below the norm of $a-a^{2}$ in $V^{\prime}$. For that purpose we recall that

$$
\left\|a-a^{2}\right\|_{V^{\prime}}=\sup _{\phi \in V, \phi \neq 0} \frac{\left|<a-a^{2}, \phi>\right|}{\|\phi\|_{V}}
$$

and use appropriate test functions $\phi$. First we take

$$
\begin{aligned}
\phi_{j}^{(j k)}\left(x_{j}\right) & \left.=\phi_{1}\left(\frac{x_{j}-\xi_{j k}}{\epsilon}\right) \text { on }\right] 0, l_{j}[, \\
\phi_{j^{\prime}}^{(j k)} & =0 \text { if } j^{\prime} \neq j,
\end{aligned}
$$

where $\phi_{1}$ is a fixed function defined by

$$
\phi_{1}(\hat{x})=\left\{\begin{array}{l}
-2(3 / 2+\hat{x}) \text { if }-3 / 2<\hat{x} \leq-1, \\
\hat{x} \text { if }-1<\hat{x} \leq 0 \\
-\hat{x} \text { if } 0 \leq \hat{x}<1 \\
2(3 / 2-\hat{x}) \text { if } 1 \leq \hat{x}<3 / 2 \\
0 \text { else. }
\end{array}\right.
$$

With this choice we have

$$
\begin{aligned}
<a-a^{2}, \phi^{(j k)}> & =\alpha_{j k} \phi^{(j k)}\left(\xi_{j k}\right)-\alpha_{j k}^{2} \phi^{(j k)}\left(\xi_{j k}^{2}\right) \\
& =\alpha_{j k}^{2}\left(\phi^{(j k)}\left(\xi_{j k}\right)-\phi^{(j k)}\left(\xi_{j k}^{2}\right)\right)
\end{aligned}
$$


since $\phi^{(j k)}\left(\xi_{j k}\right)=0$. By the finite increment theorem and the fact that $\left|\xi_{j k}-\xi_{j k}^{2}\right|<\epsilon$, we then obtain

$$
\left|<a-a^{2}, \phi^{(j k)}>\right|=\frac{\left|\alpha_{j k}^{2}\right|}{\epsilon}\left|\xi_{j k}-\xi_{j k}^{2}\right| .
$$

This estimate yields

$$
\left|\alpha_{j k}^{2}\right|\left|\xi_{j k}-\xi_{j k}^{2}\right| \leq \epsilon\left|<a-a^{2}, \phi^{(j k)}>\right| \leq \epsilon\left\|a-a^{2}\right\|_{V^{\prime}}\left\|\phi^{(j k)}\right\|_{V},
$$

and leads to

$$
\left|\alpha_{j k}^{2}\right|\left|\xi_{j k}-\xi_{j k}^{2}\right| \leq C \sqrt{\epsilon}\left\|a-a^{2}\right\|_{V^{\prime}},
$$

for some positive constant $C$ since one readily checks that $\left\|\phi^{(j k)}\right\|_{V}=\frac{C}{\sqrt{\epsilon}}$.

From the fourth assumption on $\epsilon$, we have

$$
\left|\alpha_{j k}^{2}\right| \geq m / 2
$$

where $m=\min _{j, k}\left|\alpha_{j k}\right|$. These two estimates finally give

$$
\left|\xi_{j k}-\xi_{j k}^{2}\right| \leq \frac{2 C \sqrt{\epsilon}}{m}\left\|a-a^{2}\right\|_{V^{\prime}} .
$$

Now we take

$$
\begin{aligned}
\phi_{j}^{(j k)}\left(x_{j}\right) & \left.=\phi_{2}\left(\frac{x_{j}-\xi_{j k}}{\delta}\right) \text { on }\right] 0, l_{j}[, \\
\phi_{j^{\prime}}^{(j k)} & =0 \quad \text { if } j^{\prime} \neq j,
\end{aligned}
$$

where $\delta=\frac{1}{2} \min _{j, k \neq k^{\prime}}\left|\xi_{j k}-\xi_{j k^{\prime}}\right|$ and $\phi_{2}$ in the form

$$
\phi_{2}(\hat{x})=\left\{\begin{array}{l}
\hat{x}+1 \text { if }-1<\hat{x} \leq 0 \\
1-\hat{x} \text { if } 0 \leq \hat{x}<1 \\
0 \text { else }
\end{array}\right.
$$

With this choice we have

$$
\begin{aligned}
<a-a^{2}, \phi^{(j k)}> & =\alpha_{j k} \phi^{(j k)}\left(\xi_{j k}\right)-\alpha_{j k}^{2} \phi^{(j k)}\left(\xi_{j k}^{2}\right) \\
& =\left(\alpha_{j k}-\alpha_{j k}^{2}\right) \phi^{(j k)}\left(\xi_{j k}\right)+\alpha_{j k}^{2}\left(\phi^{(j k)}\left(\xi_{j k}\right)-\phi^{(j k)}\left(\xi_{j k}^{2}\right)\right), \\
& =\left(\alpha_{j k}-\alpha_{j k}^{2}\right)+\alpha_{j k}^{2}\left(\phi^{(j k)}\left(\xi_{j k}\right)-\phi^{(j k)}\left(\xi_{j k}^{2}\right)\right) .
\end{aligned}
$$

Therefore by the finite increment theorem we obtain as before

$$
\left|\alpha_{j k}-\alpha_{j k}^{2}\right| \leq\left|<a-a^{2}, \phi^{(j k)}>\right|+\frac{1}{\delta}\left|\alpha_{j k}^{2}\right|\left|\xi_{j k}-\xi_{j k}^{2}\right|,
$$


and by the estimate (48) we get

$$
\left|\alpha_{j k}-\alpha_{j k}^{2}\right| \leq\left|<a-a^{2}, \phi^{(j k)}>\right|+\frac{C \sqrt{\epsilon}}{\delta}\left\|a-a^{2}\right\|_{V^{\prime}} .
$$

Since $\left\|\phi^{(j k)}\right\|_{V}=\frac{C_{1}}{\sqrt{\delta}}$ for some $C_{1}>0$, we have obtained

$$
\left|\alpha_{j k}-\alpha_{j k}^{2}\right| \leq\left(\frac{C_{1}}{\sqrt{\delta}}+\frac{C \sqrt{\epsilon}}{\delta}\right)\left\|a-a^{2}\right\|_{V^{\prime}}
$$

The estimates (47), (49) and (50) lead to the conclusion.

In the above theorem if like in [10] we are only interested in the stability of the locations of the point sources, i.e. if we assume that $\alpha_{j k}^{2}=\alpha_{j k}$, then we can obtain a more accurate estimate under less assumptions on $\epsilon$, namely we have the

Theorem 5.2. Fix one exterior vertex $E_{i_{0}}$ of $G$ and $T>T_{0}$ with $T_{0}>0$ from Lemma 3.3. Suppose that $A^{2}=\left(\left(\alpha_{j k}, \xi_{j k}^{2}\right)_{k=1}^{K_{j}}\right)_{j=1}^{N}$ is in $\Sigma \cap B(A, \epsilon)$ with $\epsilon>0$ satisfying (43) to (45). Then there exists a constant $C$ depending on $T, \min _{j, k \neq k^{\prime}}\left|\xi_{j k}-\xi_{j k^{\prime}}\right|$ and $\min _{j, k}\left|\alpha_{j k}\right|$ such that

$$
\sum_{j=1}^{N} \sum_{k=1}^{K_{j}}\left|\xi_{j k}-\xi_{j k}^{2}\right| \leq C \sqrt{\epsilon} \sum_{i \in I_{\text {ext }} \backslash\left\{i_{0}\right\}}\left\|u_{j_{i}}^{\prime}\left(E_{i}, t\right)-\left(u^{2}\right)_{j_{i}}^{\prime}\left(E_{i}, t\right)\right\|_{L^{2}(0, T)} .
$$

Proof. It suffices to take

$$
\begin{array}{rll}
\phi_{j}^{(j k)}\left(x_{j}\right) & \left.=\phi_{2}\left(\frac{x_{j}-\xi_{j k}}{\epsilon}\right) \text { on }\right] 0, l_{j}[, \\
\phi_{j^{\prime}}^{(j k)} & =0 & \text { if } j^{\prime} \neq j
\end{array}
$$

with the same $\phi_{2}$ as before and use the above arguments.

Remark 5.3. The constraints (44) and (45) could be suppressed but this requires choices of more tricky test functions $\phi^{(j k)}$ and will modify the constants in the estimates of Theorems 5.1 and 5.2. We do not treat these cases for the sake of simplicity.

\section{Reconstruction}

For the reconstruction of the point sources from boundary measurements we follow the point of view of [36] which consists in using the following exact controllability result: 
Lemma 6.1. Fix one exterior vertex $E_{i_{0}}$ of $G$ and $T>T_{0}$ with $T_{0}>0$ from Lemma 3.3. Then for every $\phi \in V$, there exist unique controls $v_{j_{i}} \in H_{0}^{1}(0, T), i \in I_{\text {ext }} \backslash\left\{i_{0}\right\}$, such that the (weak) solution $\psi \in C([0, T] ; H) \cap C^{1}\left([0, T] ; V^{\prime}\right)$ of

$$
\left\{\begin{array}{l}
\partial_{t}^{2} \psi_{j}\left(x_{j}, t\right)-\frac{d_{j}}{m_{j}} \psi_{j}{ }^{\prime}\left(x_{j}, t\right)=0 \text { in } Q_{j T}, \forall j=1, \cdots, N, \\
\psi(\cdot, t)=0 \text { is continuous on } G \text { for all } t \in] 0, T[ \\
\left.\sum_{j \in N_{i}} d_{j} \frac{\partial \psi_{j}}{\partial \nu_{j}}\left(E_{i}, t\right)=0, \forall i \in I_{i n t}, \forall t \in\right] 0, T[ \\
\left.\psi_{j_{i}}\left(E_{i}, t\right)=v_{j_{i}}, \forall i \in I_{e x t} \backslash\left\{i_{0}\right\}, \forall t \in\right] 0, T[ \\
\left.\psi_{j_{0}}\left(E_{i_{0}}, t\right)=0, \forall t \in\right] 0, T[ \\
\left.\psi_{j}\left(x_{j}, 0\right)=\phi_{j}\left(x_{j}\right), \partial_{t} \psi_{j}\left(x_{j}, 0\right)=0 \text { in }\right] 0, l_{j}[, \forall j=1, \cdots, N
\end{array}\right.
$$

satisfies

$$
\psi(\cdot, T)=\partial_{t} \psi(\cdot, T)=0
$$

Proof. This is a direct consequence of Lemmas 3.2, 3.3 and 3.4 and of the Hilbert Uniqueness Method of Lions [26, Th. I.6.4] (see for instance [34, 24, 18] for such applications to networks). Note that $\psi$ is only a weak solution of the system (51) with the final conditions (52) in the sense that $\psi$ is the unique solution of (using the transposition method)

$$
\begin{aligned}
\sum_{j=1}^{N} m_{j} \int_{Q_{j} T} \psi_{j} f_{j} d x d t & =-<\partial_{t} \varphi(0), \phi>_{V^{\prime}-V} \\
& -\sum_{i \in I_{e x t} \backslash\left\{i_{0}\right\}} d_{j_{i}} \nu_{j_{i}}\left(E_{i}\right)<\varphi_{j_{i}}^{\prime}\left(E_{i}, \cdot\right), v_{j_{i}}>_{H^{-1}(0, T)-H_{0}^{1}(0, T)}
\end{aligned}
$$

for all $f \in L^{1}(0, T ; H), \varphi_{0} \in H, \varphi_{1} \in V^{\prime}$, where $\varphi \in C([0, T] ; H) \cap C^{1}\left([0, T] ; V^{\prime}\right)$ is the unique solution of (whose existence follows from Lemma 3.4 )

$$
\left\{\begin{array}{l}
\left.\partial_{t}^{2} \varphi=A \varphi+f \text { in }\right] 0, T[ \\
\varphi(T)=\varphi_{0}, \partial_{t} \varphi(T)=\varphi_{1}
\end{array}\right.
$$

In view of Lemma 6.1 we can define a bounded linear operator $\Pi: V \rightarrow H_{0}^{1}(0, T)^{M}$, by

$$
\phi \rightarrow\left(v_{j_{i}}\right)_{i \in I_{e x t} \backslash\left\{i_{0}\right\}},
$$

where $M$ is the cardinal of $I_{e x t} \backslash\left\{i_{0}\right\}$ and $v_{j_{i}}$ are the controls from the above Theorem driving the system (51) to rest at time $T$. 
We further use the adjoint $K_{L^{2}}^{\star}$ of the operator $K$ as (bounded) operator from $L^{2}(0, T)$ into itself and which is given by (see section 6 of [36])

$$
\left(K_{L^{2}}^{\star} \eta\right)(t)=\int_{t}^{T} \lambda(s-t) \eta(s) d s, 0<t<T
$$

for all $\eta \in L^{2}(0, T)$. By the assumption (2) we even have (see section 6 of [36])

$$
R\left(K_{L^{2}}^{\star}\right)={ }^{0} H^{1}(0, T) .
$$

Consequently for all $\psi \in{ }^{0} H^{1}(0, T)$ there exists a unique $\eta \in L^{2}(0, T)$ solution of (since $\operatorname{ker} K_{L^{2}}^{\star}=R(K)^{\perp}=\{0\}$ )

$$
K_{L^{2}}^{\star} \eta=\psi
$$

equivalently, $\eta$ is solution of the Volterra equation of the first kind

$$
\int_{t}^{T} \lambda(s-t) \eta(s) d s=\psi(t), 0<t<T
$$

We then define the mapping $\Phi$ from ${ }^{0} H^{1}(0, T)$ to $L^{2}(0, T)$ by

$$
\psi \rightarrow \eta:=\Phi \psi
$$

when $\eta$ is solution of the above integral equation. This means that

$$
K_{L^{2}}^{\star} \Phi=I d \text { on }{ }^{0} H^{1}(0, T) .
$$

Now we can formulate our reconstruction result:

Theorem 6.2. Fix one exterior vertex $E_{i_{0}}$ of $G$ and $T>T_{0}$ with $T_{0}>0$ from Lemma 3.3. For all $k=1, \cdots, \infty$ we define

$$
\theta_{k}=\Phi \Pi \phi_{k}
$$

Let $u \in C([0, T] ; V) \cap C^{1}([0, T] ; H)$ be the unique solution of (11) with datum a in the form (12). Then for all $k=1, \cdots, \infty$ we have

$$
<a, \phi_{k}>=-\sum_{i \in I_{e x t} \backslash\left\{i_{0}\right\}} d_{j_{i}} \nu_{j_{i}}\left(E_{i}\right)\left(u_{j_{i}}^{\prime}\left(E_{i}, \cdot\right),\left(\theta_{k}\right)_{j_{i}}\right)_{L^{2}(0, T)},
$$

and then a may be reconstructed by

$$
a=\sum_{k=1}^{\infty}<a, \phi_{k}>\phi_{k}=-\sum_{k=1}^{\infty}\left(\sum_{i \in I_{\text {ext }} \backslash\left\{i_{0}\right\}} d_{j_{i}} \nu_{j_{i}}\left(E_{i}\right)\left(u_{j_{i}}^{\prime}\left(E_{i}, \cdot\right),\left(\theta_{k}\right)_{j_{i}}\right)_{L^{2}(0, T)}\right) \phi_{k} .
$$


Proof. Applying the identity (53) with $\varphi=v$, where $v$ is the unique solution of (28) with initial speed $a$ we have:

$$
<a, \phi_{k}>=-\sum_{i \in I_{\text {ext }} \backslash\left\{i_{0}\right\}} d_{j_{i}} \nu_{j_{i}}\left(E_{i}\right)<v_{j_{i}}^{\prime}\left(E_{i}, \cdot\right),\left(\Pi \phi_{k}\right)_{j_{i}}>_{H^{-1}(0, T)-H_{0}^{1}(0, T)} .
$$

To conclude we need to show that

$$
<v_{j_{i}}^{\prime}\left(E_{i}, \cdot\right),\left(\Pi \phi_{k}\right)_{j_{i}}>_{H^{-1}(0, T)-H_{0}^{1}(0, T)}=\left(u_{j_{i}}^{\prime}\left(E_{i}, \cdot\right),\left(\theta_{k}\right)_{j_{i}}\right)_{L^{2}(0, T)} .
$$

Let us first prove that there exists $h_{j_{i}} \in H_{-1}(0, T)$ such that

$$
u_{j_{i}}^{\prime}\left(E_{i}, \cdot\right)=K h_{j_{i}}
$$

and satisfying

$$
<v_{j_{i}}^{\prime}\left(E_{i}, \cdot\right), \chi>_{H^{-1}(0, T)-H_{0}^{1}(0, T)}=<h_{j_{i}}, \chi>_{H_{-1}(0, T)-{ }^{0} H^{1}(0, T)}, \forall \chi \in H_{0}^{1}(0, T) .
$$

Indeed the identity (58) clearly follows from Lemmas 3.5 and 4.2 ; moreover using an approximation sequence of $a_{n}$ as usual, the corresponding $u_{n}$ and $v_{n}$ satisfy

$$
v_{n j_{i}}^{\prime}\left(E_{i}, \cdot\right) \rightarrow h_{j_{i}} \text { in } H_{-1}(0, T) \text {, as } n \rightarrow \infty,
$$

due to Lemmas 3.5 and 4.2 , while by Lemma 3.4 we have

$$
v_{n j_{i}}^{\prime}\left(E_{i}, \cdot\right) \rightarrow v_{j_{i}}^{\prime}\left(E_{i}, \cdot\right) \text { in } H^{-1}(0, T), \text { as } n \rightarrow \infty .
$$

The identity (59) then follows from the two above convergence properties and the continuity of the mapping $I d^{\star}$ from $H_{-1}(0, T)$ into $H^{-1}(0, T)$.

Now by the definition of $\theta_{k}$ and (54) we may write

$$
K_{L^{2}}^{\star} \theta_{k}=K_{L^{2}}^{\star} \Phi \Pi \phi_{k}=\Pi \phi_{k} .
$$

Therefore using (59) and the above identity, the left-hand side of (57) may be transformed as follows

$$
\begin{aligned}
<v_{j_{i}}^{\prime}\left(E_{i}, \cdot\right),\left(\Pi \phi_{k}\right)_{j_{i}}>_{H^{-1}(0, T)-H_{0}^{1}(0, T)}= & <h_{j_{i}},\left(\Pi \phi_{k}\right)_{j_{i}}>_{H_{-1}(0, T)-{ }^{0} H^{1}(0, T)} \\
= & <h_{j_{i}}, K_{L^{2}}^{\star}\left(\theta_{k}\right)_{j_{i}}>_{H_{-1}(0, T)-{ }^{0} H^{1}(0, T)},
\end{aligned}
$$

and from the embeddings ${ }^{0} H^{1}(0, T) \hookrightarrow L^{2}(0, T) \hookrightarrow H_{-1}(0, T)$, we get

$$
<h_{j_{i}}, K_{L^{2}}^{\star}\left(\theta_{k}\right)_{j_{i}}>_{H_{-1}(0, T)-{ }^{0} H^{1}(0, T)}=\left(K h_{j_{i}},\left(\theta_{k}\right)_{j_{i}}\right)_{L^{2}(0, T)} .
$$

This proves (57) since the above right-hand side coincides with the right-hand side of (57) due to (58). 
Remark 6.3. In [10] Bruckner and Yamamoto use another method for the reconstruction of point sources in the real interval ]0,1[. This method consists in solving a finite system of nonlinear equations but it relies on the fact that the series

$$
\left.\sum_{k=1}^{\infty} \frac{\phi_{k}(x) \phi_{k}(\xi)}{\lambda_{k}}, \text { for } x, \xi \in\right] 0,1[
$$

is differentiable in $x \in[0, \xi]$, this differentiability property being proved by computing explicitly the above series by Fourier analysis, which is possible since $\lambda_{k}=k^{2} \pi^{2}$ and $\phi_{k}(x)=\sqrt{2} \sin (k \pi x)$. In our case the calculation of the above series as well as its differentiability cannot be easily obtained since the eigenvalues and eigenvectors are not explicitly known and since $\lambda_{k}$ behaves like $k^{2}$ (the series of the derivatives being not absolutely convergent). For the real interval ]0, 1[, our method gives an alternative way to reconstruct the source $a$ without any restriction on $a$, i.e., for any values of the parameters in (12). The only drawback is that $a$ is obtained as a Fourier series which may converge slowly.

\section{References}

[1] K. Aki and P. G. Richards, Quantitative seismology theory and methods, Vol. 1, Freeman, New York, 1980.

[2] F. Ali Mehmeti, A characterisation of generalized $C^{\infty}$ notion on nets, Integral Eq. and Operator Theory, 9, 1986, 753-766.

[3] F. Ali Mehmeti, Regular solutions of transmission and interaction problems for wave equations, Math. Meth. Appl. Sc., 11, 1989, 665-685.

[4] F. Ali Mehmeti, Nonlinear wave in networks, Math. Res. 80, Akademie Verlag, 1994.

[5] J. von Below, A characteristic equation associated to an eigenvalue problem on $c^{2}$ networks, Linear Algebra and appl. 71, 1985, 309-325.

[6] J. von Below, Classical solvability of linear parabolic equations on networks, J. Differential Equation, 72, 1988, 316 - 337.

[7] J. von Below, Sturm-Liouville eigenvalue problems on networks, Math. Meth. Appl. Sc., 10, 1988, 383-395.

[8] J. von Below, Parabolic Network Equations, Habilitation Thesis, Eberhard-KarlsUniversität Tübingen, 1993.

[9] G. Bruckner and M. Yamamoto, Identification of point sources by boundary observations: uniqueness, stability and reconstruction, in: E. A. Lipitakis ed., Hermis' 96, Proceedings of the third hellenic european conference on Math. and Informatics, Athens, LEA, 154$161,1997$.

[10] G. Bruckner and M. Yamamoto, On the determination of point sources by boundary observations: uniqueness, stability and reconstruction, Preprint WIAS, 252, Berlin, 1996.

[11] G. Bruckner and M. Yamamoto, Determination of point wave sources by pointwise observations: stability and reconstruction, Inverse problems, 16, 2000, 723-748. 
[12] A. Borovskikh, R. Mustafokulov, K. Lazarev and Yu. Pokornyi, A class of fourth-order differential equations on a spatial net, Doklady Math., 52, 1995, 433-435.

[13] G. Chen, M. Delfour, A. Krall and G. Payre, Modelling, stabilization and control of serially connected beams, Siam J. Control and Opt., 25, 1987, 526-546.

[14] G. Chen, S. Krantz, D. Russell, C. Wayne, H. West and M. Coleman, Analysis, design, and behavior of dissipative joints for coupled beams, Siam J. Appl. Math., 49, 1989, 1665-1693.

[15] G. Chen and J. Zhou, The wave propagation method for the analysis of boudary stabilization in vibrating structures, Siam J. Appl. Math., 50, 1990, 1254-1283.

[16] P.G. Ciarlet, H. Le Dret and R. Nzengwa, Junctions between three-dimension and twodimensional linearly elastic structures, J. Math. Pures et Appl., 68, 1989, 261-295.

[17] F. Conrad, Stabilization of vibrating beams by a specific feedback, in: A. V. Balakrishnan and J. P. Zolésio eds., Stabilization of flexible structures, Opt. Software Inc., 1988, 36-51.

[18] R. Dager and E. Zuazua, Controllability of tree-shaped networks of vibrating strings, C. R. Acad. Sci. Paris, 332, Série I, 2001, 1087-1092.

[19] P. Grisvard, Elliptic problems in nonsmooth domains. Pitman, Boston, 1985.

[20] T. Kato, Perturbation theory for linear operators, Springer-Verlag, 1966.

[21] J. E. Lagnese, Modeling and controllability of Plate-Beam systems, J. Math. Systems, Estimation and Control., 5, 1995, 141-187.

[22] J. E. Lagnese, G. Leugering and E.J.P.G. Schmidt, Modeling of dynamic networks of thin thermoelastic beams, Math. Meth.in the Appl. Sci., 16, 1993, 327-358.

[23] J. E. Lagnese, G. Leugering and E.J.P.G. Schmidt, Control of planar networks of Timoshenko beams, SIAM J. Control Optim., 31, 1993, 780-811.

[24] J. E. Lagnese, G. Leugering and E.J.P.G. Schmidt, Modeling, analysis and control of dynamic elastic multi-link structures, Birkhäuser, Boston, 1994.

[25] H. Le Dret, Problèmes variationnels dans les multi-domaines. Modélisation des jonctions et applications, RMA 19, Masson, Paris, 1991.

[26] J.-L. Lions, Contrôlabilité exacte, perturbations et stabilisation de systèmes distribués, tome 1, RMA 8, Masson, Paris, 1988.

[27] S. Nicaise, Some results on spectral theory over networks applied to nerve impulse transmission, Lecture Notes in Math., 1171, Springer-Verlag, 1985, 532-541.

[28] S. Nicaise, Diffusion sur les espaces ramifiés, Thesis, Université de Mons, 1986.

[29] S. Nicaise, Spectre des réseaux topologiques finis, Bull. Sc. Math., 2ème série, 111, 1987, 401-413.

[30] S. Nicaise, Exact controllability of a pluridimensional coupled problem, Revista Matematica Univ. Complutense Madrid, 5, 1992, 91-135.

[31] S. Nicaise, About the Lamé system in a polygonal or a polyhedral domain and a coupled problem between the Lamé system and the plate equation II : Exact controllability, Ann. Scuola Normale Sup. Pisa, Series IV, 20, 1993, 163-191. 
[32] S. Nicaise, Boundary exact controllability of interface problems with singularities II: Addition of internal controls, SIAM J. Control Optim., 35, 1997, p. 585-603.

[33] J.P. Puel and E. Zuazua, Exact controllability for a model of multidimensional flexible structure, Proc. Royal Soc. Edinburgh, 123 A, 1993, 323-344.

[34] E.J.P.G. Schmidt, On the modelling and exact controllability of networks of vibrating strings, SIAM J. Control Optim., 30, 1992, 229-245.

[35] M. Yamamoto, Well-posedness of an inverse hyperbolic problem by the Hilbert uniqueness method, J. Inverse and Ill-Problems, 2, 1994, 349-368.

[36] M. Yamamoto, Stability, reconstruction and regularization for an inverse source hyperbolic problem by a control method, Inverse Problems, 11, 1995, 481-496.

[37] M. Yamamoto, Determination of forces in vibrations of beams and plates by pointwise and line observations, J. Inverse and Ill-Problems, 4, 1996, 437-457. 